Kalpa Publications in Engineering
Volume 1, 2017, Pages 387-393
$\begin{aligned} & \text { ICRISET2017. International Conference on Re- } \\ & \text { search and Innovations in Science, Engineering } \\ & \text { \&Technology. Selected Papers in Engineering }\end{aligned}$
Engine ring

\title{
Compressive Sensing for Natural Images
}

\author{
Mayur Sevak ${ }^{1}$,Anish Bagga ${ }^{2}$,Arpita Agrawal ${ }^{3}$, Krusha Jani $^{4}$ \\ ${ }^{1}$ Assistant Professor, Electronics \& Communication department, B.V.M Engineering College \\ ${ }^{2,3,4}$ final year students, Electronics \& Communication department,B.V.M Engineering College
}

\begin{abstract}
The Compressive sensing technique is a new era of arising platform for signal processing and data acquisition. The significant statement of Compressive sensing is that recovery of certain images or signals from fewer samples than required. On the encoding side two properties of a signal is required that are incoherence and sparsity. Initially, the signal is converted into specific transform i.e. wavelet using sensing matrix it takes required coefficients that has less dimensionality than the image dimensions and thereby, we get resultant matrix which is also called as measurements which in turn are non-adaptive. Similarly, on the decoding side, due to low dimension of transmitted vector matrix, convex optimization is required to solve this problem. Convex optimization (L1 minimization) provides an answer to undetermined linear systems without the knowledge of nature of undergoing parameters through the systems.

In this paper, the work is being done on natural image compression. Compression of various black and white natural images is being done with help of 'haar wavelet' with second level decomposition and also reconstruct the same with three measurements of $60 \%, 70 \%$ and $80 \%$. Also we have measured same with PSNR (Peak Signal to Noise Ratio), RMSE (Root Mean Square Error), CoC (Correlation Coefficients) of them.
\end{abstract}

\section{Introduction}

Digital signal processing and in particular image processing having been developed over many years are used as the recent advancements in computing technology have made it possible to use Image Processing in day-to-day applications. The new challenge is the increasing use of images and user's expectation of the images to be transmitted in minimum of time and taking up little storage as much as possible. These requirements call for efficient image compression algorithm. The user wants efficient decompression with compression. However, the degree of compression and amount of data lost are of much significance which calls for a method combining high compression ratio with low amount of data loss.Since, data sequences or practical images normally contain significant amount of redundancy in signal or in other words correlation with neighboring signal values. A data sequence which has 
redundancy, can be compactly represented if redundancy is removed by suitable transformation methods. Popular transforms used are Discrete Cosine Transform, Discrete wavelet transform, Fast Fourier Transform etc. Compressive Sensing technique in which recovery is done from fewer samples than originally required. It utilizes the fact that sampling a signal below Nyquist rate is possible and it is analogous to number of samples to be encoded or transmitted. On encoder side initially, image is first converted into one dimensional vector with use of a wavelet transform. After transforming it is checked for incoherence [1], sparsity and RIP (Restricted Isometry Property)[1] and then with the help of random projection it is being transmitted. Dimension of transmission vector is low as compared with original one dimensional vector. On Decoder side convex optimization is used to decode the transmitted vector and then it is processed through which samples are reconstructed. And finally inverse transform is taken of this vector to reproduce the main image.Compressive Sensing

\section{Compressed Sensing}

The basic image compression and data acquisition system is known as Compressive Sensing (CS) and acquires image from the original image from less number of samples. To do that, firstly an image is converted into a vector having single dimensionality of Nx1. Then we get the resultant matrix having dimensionality of Mx1 with support of sensing matrix having dimensionality of $\mathrm{MxN}$. This is also known as M measurements. Convex optimization is used on the decoding side for reconstruction of the image as it gives a better solution to the undetermined linear systemwithout having much idea about the system parameters. As because from study L1 minimizationis better than L0 and L2, we have used L1 minimization for reconstruction 




Figure 1 : Compressed Sensing Flow 


\subsection{Sparsity}

Sparsity is defined as the degree of freedom or randomness in a signal and is smaller than the finite signal length. The fact that natural signals which are sparse in nature when expressed in proper basis function $\Psi[1]$ having a concise presentation is denied. If $f \in R n$ such that which we expand in ortho-normal basis $\Psi$, then

$$
f(t)=\Sigma x i \Psi i(t)
$$

where $\mathrm{x}$ is co-efficient sequence of ' $\mathrm{f}$ '. So from this one can discard small magnitude coefficients and keep only large value coefficients.

\subsection{Incoherence}

It signifies the idea of duality between frequency and time domain. The object of sparse representation is spread out in the same domain of their acquired domain i.e. spike or Dirac in time domain is spread out in frequency domain. A pair of ortho-basis $(\Phi, \Psi)$. Where $\Phi$ is used to sense $f$ and $\Psi$ is used to represent $f$. The coherence of these two parameters is given by.

$$
\mu(\Phi, \Psi)=\sqrt{n} \max |<\Phi, \Psi>|
$$

\subsection{L1 Minimization}

The Proposed reconstruction of $\mathrm{f}^{\wedge}$ is given by $\mathrm{f}^{\wedge}=\psi \mathrm{x}^{\wedge}$ where $\mathrm{x}^{\wedge}$ is solution to convex optimization program. L1 norm minimization is given as

$$
\|\mathrm{x}\|_{1}=\Sigma|\mathrm{xi}| \text {, where i from } 1 \text { to } \mathrm{n}
$$

So for reconstruction faithfully it is subject to minimize the L1 norm. The use of L1 norm minimization is to recover the sparse signals. Greedy algorithm have also been proposed for reconstruction.

\section{Wavelet Transform}

For effective analysis of image, it is the mathematical tool used to manipulate the signal (or image). Wavelet Transform makes it easier for compression, transmission and analysis of many images. Fourier and wavelet transform both are based on basis function. For bifurcation of information of an image into an approximation and detailed sub signals, Wavelet Analysis can be used. The general trend of pixel values is shown by approximation and the vertical, horizontal and diagonal details/ changes in the image is shown by detailed sub signal. For small value of details, the value can be approximated to zero. Threshold is the value after which the data is considered small enough to be set to zero. The higher the number of zeros the higher the compression that can be achieved. 


\subsection{Haar Wavelet}

The simplest type of wavelet is HAAR Wavelet. Wavelet can keep track of time and frequency information. Haar wavelet compression is an efficient way to perform both lossless and lossy image compression. It relies on averaging and differencing values in an image matrix to produce a matrix which is sparse. The Haar wavelet is a sequence of rescaled "square-shaped" functions which altogether form a wavelet family.



Figure 2 :Haar Wavelet

\section{Performance parameters}

The performance or the quality of image is checked using RMSE, PSNR and CoC using the following formulas:

\section{Mean Square Error (MSE):}

If compression is lossy than it is convenient to be able to quantify the difference between original image and reconstructed image and popular choice is MSE. In the MSE, measurement the total squared difference between original and the reconstructed one is averaged over the entire signal.

$$
\begin{aligned}
& \mathrm{MSE}=\frac{1}{N M} \sum_{x=0}^{N-1} \sum_{y=0}^{M-1}\left[\left|f(x, y)-f^{\wedge}(x, y)\right|^{2}\right. \\
& R M S E=S Q R T(M S E)
\end{aligned}
$$

\section{Correlation Coefficient (CoC):}

It measures the correlation between original image and reconstructed image.

$$
\mathrm{Coc}=\frac{\sum_{x=0}^{N-1} \sum_{y=0}^{M-1}\left[\left|f(x, y)-f^{\wedge}(x, y)\right|^{2}\right.}{\sqrt{\sum_{x=0}^{N-1} \sum_{y=0}^{M-1}|f(x, y)|^{2} \sqrt{\sum_{x=0}^{N-1} \sum_{y=0}^{M-1}\left|f^{\wedge}(x, y)\right|^{2}}}}
$$




\section{Peak Signal to Noise Ratio:}

PSNR relates the MSE to maximum amplitude of original signal, this makes the measurement of the range of data.

$$
\mathrm{PSNR}=10 \log \left[\frac{255^{2}}{M S E}\right]
$$

\section{Simulation Results}

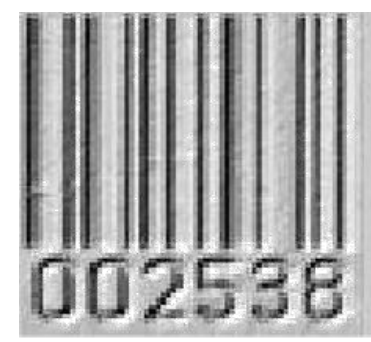

Figure 3: CS Image 1

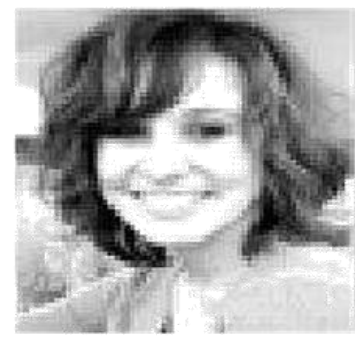

Figure 5: CS Image 2

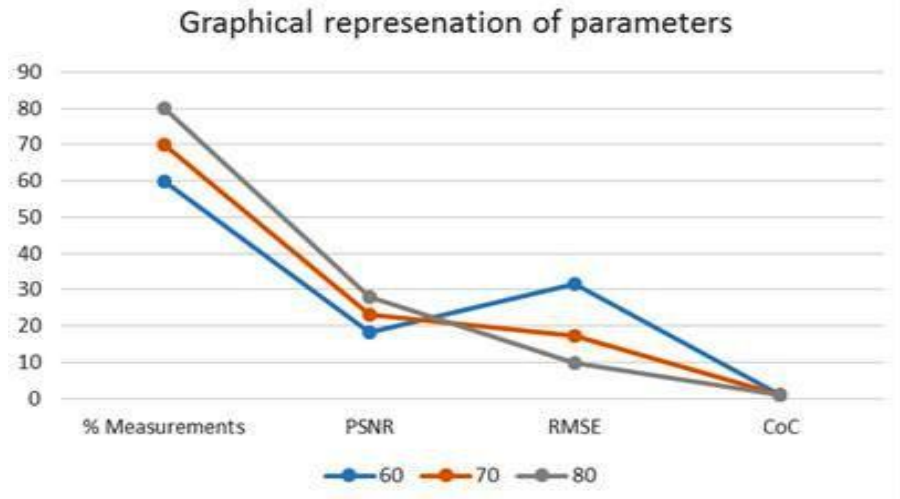

Figure 4: Comparison of $60 \%, 70 \%$ and $80 \%$ of sampled values of CS Image 1

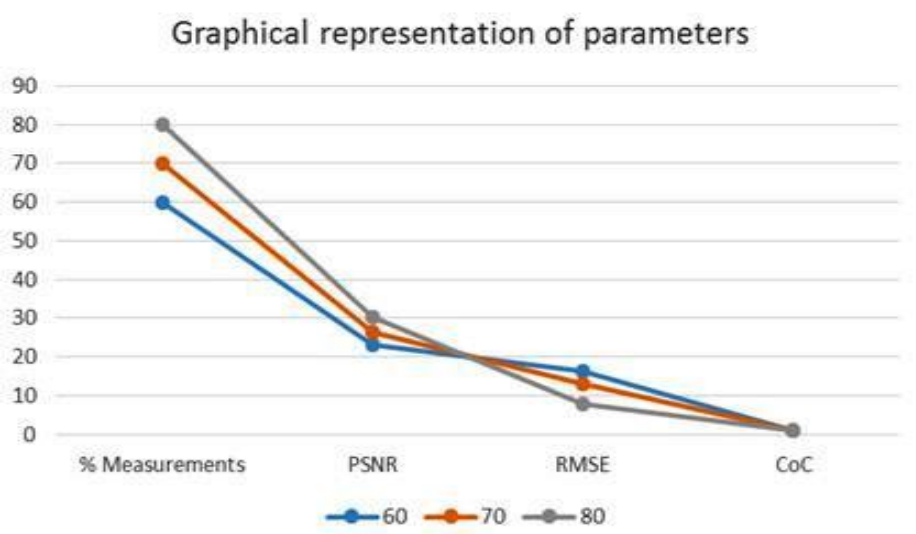

Figure 6: Comparison of $60 \%, 70 \%$ and $80 \%$ of sampled values of CS Image 2 


\section{Conclusion}

The new challenge today is that images are increasingly been used and so need to be transferred in less amount of time and to consume little space storage. This requirements call for efficient image compression algorithm. On one side, the other image compression techniques like the JPEG sense all the pixels of the image. But on the other side, compressive sensing compresses the image at the time of sensing by selecting only sparse component with the help of which the image can be recovered using convex optimization technique (L1 minimization). With the help of haar wavelet here, we have applied $\mathrm{CS}$ on natural images. We have successfully recovered the images using convex optimization technique. By comparing the parameters like PSNR, RMSE and CoC, the ability of CS to recover the images has been measured. We have also measured that as the samples increase, PSNR increases, RMSE decreases and $\mathrm{CoC}$ also increases

\section{References}

1. Marco F. Duarte, Richard G. Baranuik "Spectral compressive sensing Applied and Computational Harmonic Analysis" July 2013, Vol.35.

2. Rick Chartrand and Valentina Staneva "Restricted Isometry Properties and nonconvex compressive sensing", VOL. 24, No. 3, May 2008.

3. Lu Gan "Block Compressed Sensing of Natural Images" $15^{\text {th }}$ National Conference on Digital Signal Processing, IEEE August 2007.

4. Petros T. Boufounos, Richard G. Baranuik" 1-Bit Compreessive sensing", Published in Information Sciences and Systems, 2008, CISS 2008. $42^{\text {nd }}$ Annual Conference

5. Saeid Sanei "On optimization of the measurement matrix for compressive sensing", IEEE signal processing Magazine, 2219-5491, IEEE April 22015.

6. Volkan Cevher, Marco F. Duarte, Chinmay Hedge "Sparse Signal Recovery Using Markov Random Fields", 1987-2017 Neutal Information Processing Systems Foundation, Inc.

7. Emmanuel Candes and Justin Romberg, "Sparsity and incoherence in compressive sampling", VOL. 3, NO. 3, April 2007

8. M.A. Davenport, M. F. Duarte, Y.C. Eldar, G. Kutynoik "Introduction to Compressed Sensing" International Conference on Advances in Computing, Control, and Telecommunication Technologies 978-0-7695-3915-7/09, 2009 IEEE.

9. Mayur M.Sevak, Darshan C.Dalwadi, Falgun Thakkar, "A Survey Of various application of Compressive Sensing Technique in Image Processing", National Conference on Embedded, Power System, Signal Processing and Communication, December 2011

10. Mayur Sevak, Darshankumar Dalwadi, Amit Choksi Ripal patel. "Compressive Sensing For CT Images: A Performance Analysis of Various Wavelets", International Journal of Graphics \& Image Processing. (Feb-2014), Vol. 4, No. 1. 